\title{
Metal-organic interface functionalization via acceptor end groups: PTCDI on coinage metals
}

\author{
Antoni Franco-Cañellas, ${ }^{1}$ Qi Wang, ${ }^{2}$ Katharina Broch, ${ }^{3}$ David A. Duncan, ${ }^{4}$ Pardeep Kumar Thakur, ${ }^{4}$ Lijia Liu, ${ }^{2}$ \\ Satoshi Kera, ${ }^{5}$ Alexander Gerlach, ${ }^{1}$ Steffen Duhm, ${ }^{2}$ and Frank Schreiber ${ }^{1, *}$ \\ ${ }^{1}$ Institut für Angewandte Physik, Universität Tübingen, Auf der Morgenstelle 10, 72076 Tübingen, Germany \\ ${ }^{2}$ Institute of Functional Nano \& Soft Materials (FUNSOM) and Jiangsu Key Laboratory for Carbon-Based Functional Materials \& Devices, \\ Soochow University, Suzhou 215123, People's Republic of China \\ ${ }^{3}$ Fritz-Haber-Institut der Max-Planck-Gesellschaft, Department of Physical Chemistry, Faradayweg 4-6, 14195 Berlin, Germany \\ ${ }^{4}$ Diamond Light Source, Harwell Science and Innovation Campus, Oxfordshire OX11 ODE, United Kingdom \\ ${ }^{5}$ Department of Photo-Molecular Science, Institute for Molecular Science, Okazaki 444-8585, Japan
}

(Received 2 March 2017; published 26 June 2017)

\begin{abstract}
We present a comprehensive study of the complex interface between perylene-3,4,9,10-tetracarboxylic diimide (PTCDI) and the (111) surfaces of the three coinage metals. The specific structural, electronic, and chemical properties of the interface rendered by the different substrate reactivities are monitored with low-energy electron diffraction (LEED), x-ray standing waves (XSW), and ultraviolet and x-ray photelectron spectroscopy (UPS and XPS). In particular, the balance between molecule-substrate and molecule-molecule interactions is considered when interpreting the core-level spectra of the different interfaces. By presenting additional adsorption distances of the unsubstituted perylene, we show that the molecular functionalization via end groups with acceptor character facilitates the charge transfer from the substrate but it is not directly responsible for the associated short adsorption distances, demonstrating that this frequently assumed correlation is not necessarily correct.
\end{abstract}

DOI: 10.1103/PhysRevMaterials.1.013001

The presence of functional groups in $\pi$-conjugated molecules not only determines the specific electronic and geometrical properties of the molecule, for instance rendering an electron donor or acceptor character, but also impacts the molecule-substrate [1,2] and the molecule-molecule interactions [3]. The latter can be enhanced by electronegative groups that facilitate a directional noncovalent bonding, such as hydrogen-bonds, of special importance in the context of surface functionalization through 2D supramolecular arrangements [3]. The effect of these interactions in binary compounds of donor-acceptor molecules [4,5] deposited on metal substrates has been of particular interest, since they strongly modify the interface electronic structure, appearing as a suitable way to tune the substrate work function [6,7]. In pure compounds though, the specific effect of moleculemolecule interactions is more elusive, in particular for the electronic properties [8-11]. In this work, we provide a detailed interpretation of the interface characteristics within the context of intermolecular versus molecule-substrate interactions by presenting a comprehensive study of perylene-3,4,9,10tetracarboxylic diimide $\left(\mathrm{C}_{24} \mathrm{H}_{10} \mathrm{O}_{4} \mathrm{~N}_{2}\right.$, PTCDI) [12,13] adsorbed on the (111) surface of the coinage metals. Specifically, the adsorption distance is measured using the x-ray standing wave (XSW) technique [14], whereas the lateral order is monitored with low-energy electron diffraction (LEED). Ultraviolet and $\mathrm{x}$-ray photoelectron spectroscopy (UPS and XPS) reveal the specific electronic and chemical properties of the interface. Using the precise description of the different PTCDImetal interfaces obtained by this multitechnique approach, we discuss in detail the core-level spectra at the (sub)monolayer regime, where we correlate the different spectral features with the specific adsorption scenarios. Finally, further comparison

\footnotetext{
*frank.schreiber@uni-tuebingen.de
}

of the vertical arrangement of the unsubstituted perylene allows us to identify the specific role of the functional groups in the adsorption distance and its correlation with interface charge transfer (CT). XSW and high-resolution XPS of PTCDI (sub)monolayers were measured at beamline I09 at the Diamond Light Source, where (sub)monolayer coverages were prepared in situ and measured in a similar configuration as in Refs. [15,16]. The UPS and multilayer XPS as well as the LEED measurements were carried out at Soochow University following the same procedure as discussed in Refs. [16,17]. Further details can be found in Ref. [18].

In contrast to well-studied perylene-3,4,9,10tetracarboxylic dianhydride (PTCDA) [2,10,19-28], PTCDI has an imide instead of an anhydride end group, which drastically influences the intermolecular arrangement. Indeed, the molecules maximize the contact between adjacent imide groups through the $\mathrm{N}-\mathrm{H} \cdots \mathrm{O}$ hydrogen-bond formation $[9,29,30]$, favoring a parallel orientation [Fig. 1(a)] as opposed to the dominating perpendicular interaction between PTCDA molecules driven by their quadrupole moments [29]. By varying the substrate reactivity (weak in gold, intermediate in silver, and relatively strong in $\mathrm{Cu}$ ) we are able to monitor how the balance between molecule-molecule and molecule-substrate interactions affects the different interface characteristics.

We start with a thorough characterization of structural and electronic properties of the interfaces. Of particular interest are $\mathrm{CT}$ effects from the substrate, which are visible through a (partial) filling of the LUMO in monolayers on metals. As seen in Fig. 1(b), this is the case for PTCDI on $\mathrm{Ag}(111)$ and on $\mathrm{Cu}(111)$ (features labeled as $\mathrm{L}^{\prime}$ ). In the first case, the partially filled LUMO is cut at the Fermi edge, which means that the PTCDI monolayer on silver becomes metallic. For copper, the LUMO filling is complete, since it appears entirely below the Fermi edge, rendering a semiconducting behavior. In contrast, the LUMO of PTCDI on Au(111) remains unfilled 


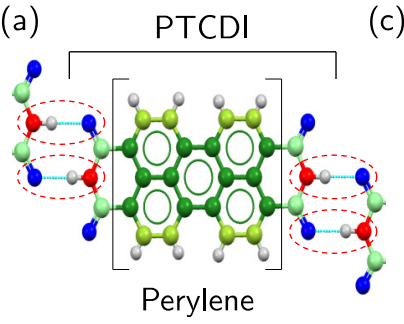

(c)

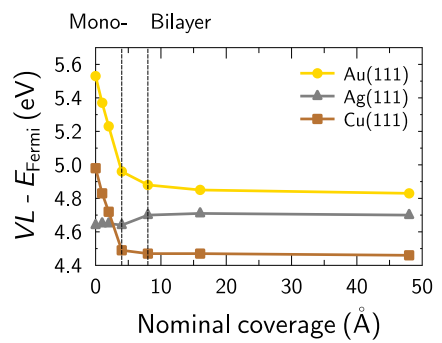

(b)

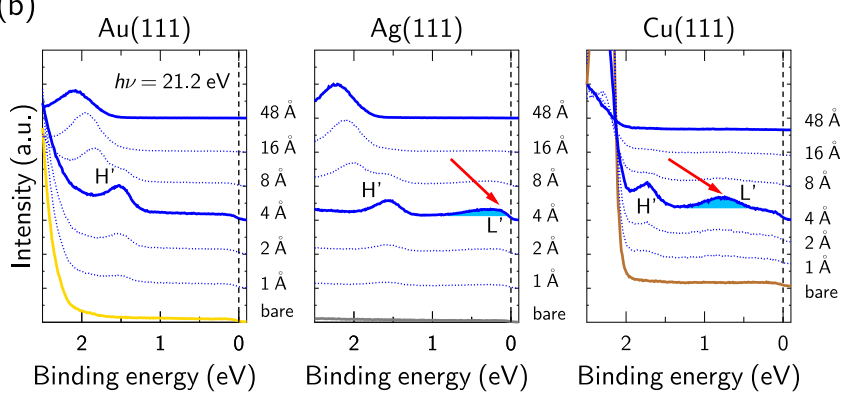

FIG. 1. (a) Sketch of PTCDI with the direction of preferential molecule-molecule interaction indicated by dashed circles $[9,29,30]$. Oxygen (blue), nitrogen (red), and inequivalent carbon atoms (green) distinguished in the XSW analysis. (b) Valence-band (VB) spectra (monochromatized He I source) for PTCDI on the different substrates. The full monolayer $(4 \AA)$ and the $\sim 10$ ML ( $48 \AA$ ) are highlighted. Filled former LUMO features are marked with an arrow and labeled with an $\mathrm{L}^{\prime}$. The relaxed HOMO is labeled with an $\mathrm{H}^{\prime}$. (c) Evolution of the vacuum-level (VL) with increasing coverage.

at any coverage. Thus we can conclude that for PTCDI the molecular coupling with the substrate increases in the order $\mathrm{Au}-\mathrm{Ag}-\mathrm{Cu}$, from physisorption to strong chemisorption, similarly to PTCDA [25]. Increasing the coverage to, nominally, two monolayers (ML) leads to a (virtually) complete attenuation of substrate features [18] indicating the formation of closed layers for (at least) the first $2 \mathrm{ML}$ of PTCDI on all three substrates. PTCDI deposition leads to interface dipoles on all substrates [Fig. 1(c)], which, on $\mathrm{Au}$, can be mainly attributed to the push-back effect [5] and on $\mathrm{Ag}$ and $\mathrm{Cu}$ to the complex interplay of push-back and charge rearrangements by the net electron transfer from the substrate to PTCDI [31]. Interestingly, the different vacuum levels (VLs) for PTCDI multilayers are in contrast to multilayer films of PTCDA on the same substrates, which show the same VL regardless of the substrate underneath [25].

The vertical adsorption geometry of PTCDI (sub)monolayers, extracted from XSW measurements (see Fig. 2) performed in back-reflection geometry using the (111) Bragg reflection of the substrates [18], is sketched in Fig. 3 together with those of unsubstituted perylene [18] and the related perylene derivatives PTCDA [22-24] and diindinoperyelene (DIP) [32] for comparison. Overall, the adsorption trend is very similar to that of PTCDA and DIP, but with some important differences stemming from the different functional groups. In particular, both oxygen and nitrogen show a strong bending on $\operatorname{Ag}(111)$, whereas on copper, only the oxygens are bent downwards and the nitrogen atoms are above the perylene core. We tentatively attribute this difference to the directional imide-imide interaction [29]

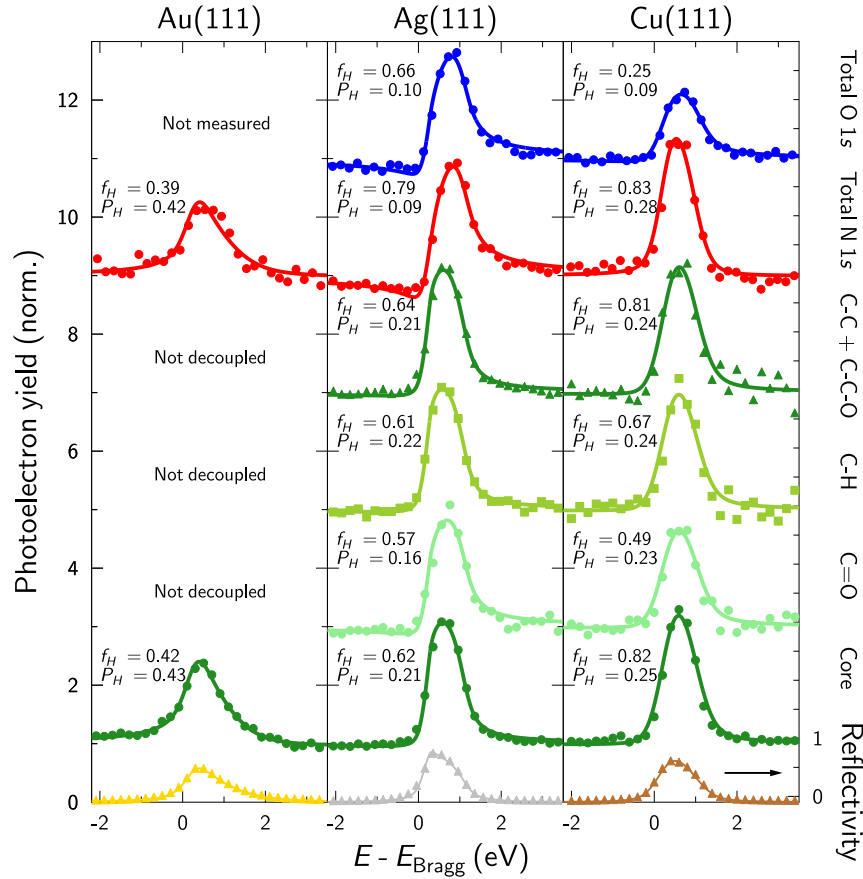

FIG. 2. XSW measurements of PTCDI (sub)monolayers adsorbed on the coinage metals. At the bottom of the figure, typical reflectivity curves for the different (111) surfaces are shown. On top, the photoelectron yield of the main components is included. The structural information is contained within the coherent position, $P_{H}$, and the coherent fraction, $f_{H}$ (see Ref. [18] for the details). The chemical sensitivity of this technique provides information for the different species, as well as for the different inequivalent carbon atoms (provided that the photoelectron yield can be successfully resolved). The curves labeled as "Core" correspond to the average adsorption distance of the $\mathrm{C}-\mathrm{C}+\mathrm{C}-\mathrm{H}+\mathrm{C}-\mathrm{C}-\mathrm{O}$ carbon species. Due to an overlapping gold Auger peak in the $\mathrm{O} 1 s \mathrm{BE}$ region the adsorption distances for this element on $\mathrm{Au}(111)$ are not accessible. Also for this substrate, the inequivalent carbon atoms could not be decoupled, thus only the average core adsorption distance is provided [18]. We note, however, the remarkable number of inequivalent carbon atoms distinguished for the copper and silver substrates.

existing on $\mathrm{Ag}(111)$, but not on $\mathrm{Cu}(111)$ (see below), which may require the alignment between oxygen and nitrogen atoms of adjacent molecules (a similar effect is expected on gold). The coherent fractions $\left(f_{H}\right)$ [18] corresponding to the carbon backbone are typical for flat-laying molecules with a significant vertical order. For comparison, unsubstituted perylene adsorbs at shorter distances, which is especially striking on $\mathrm{Cu}(111)$ as often a decreased bonding distance is associated with increased adsorbate/substrate interaction $[1,2,5]$. However, in contrast to PTCDI (and also PTCDA [25] and DIP [33]), for perylene, no (partially) filled LUMO in the monolayer on $\mathrm{Cu}(111)$ could be evidenced by UPS [34].

Turning to the lateral ordering, LEED measurements for nominal monolayer coverages (see Fig. 4) only show diffraction patterns for gold (in line with real-space studies employing scanning tunneling microscopy (STM) $[30,35,36]$ ) and silver. It can be argued that the strong bonding with the substrate prevents the long-range rearrangement of the PTCDI molecules on copper, thus hindering a supramolecular 

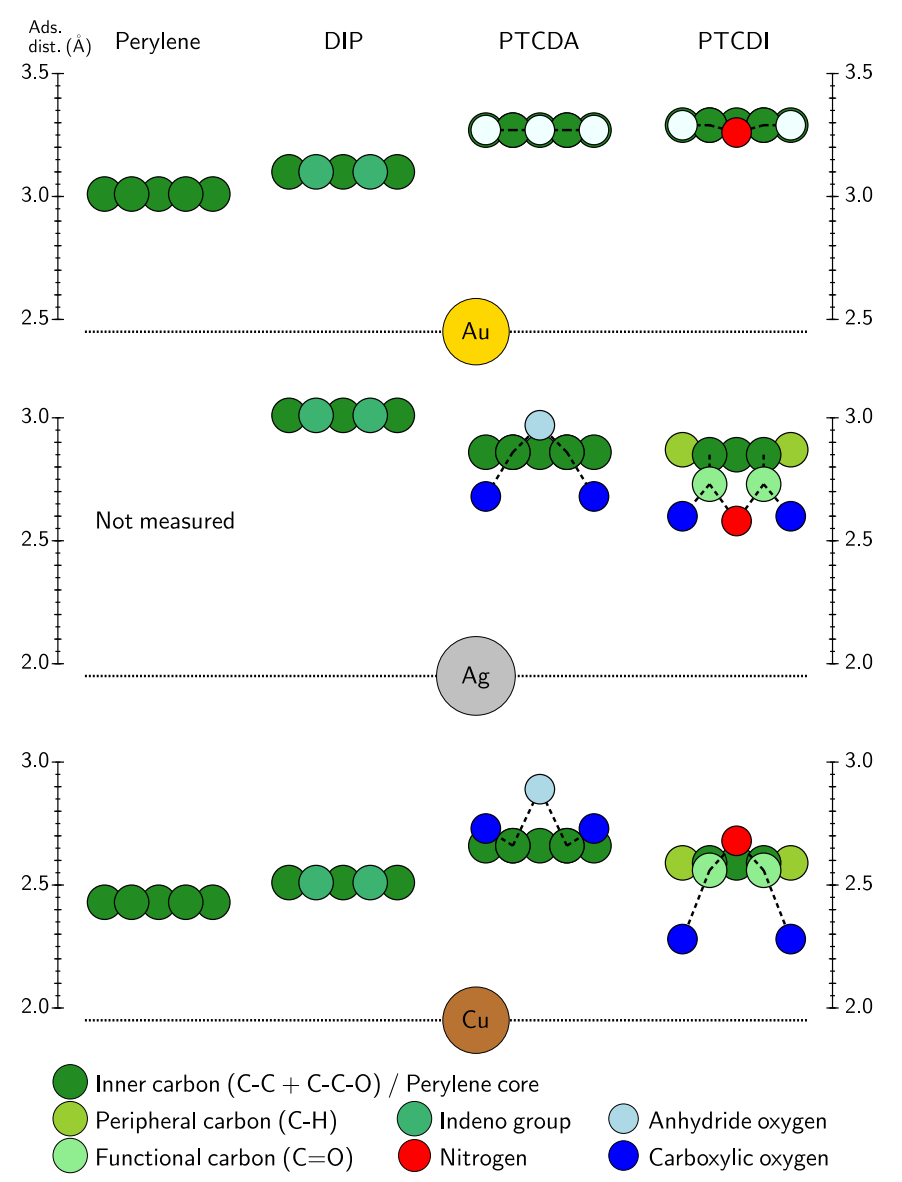

FIG. 3. Adsorption distances ( $\mathrm{A})$, extracted from XSW measurements, for different perylene derivatives. Together with the data for perylene and PTCDI, first reported in this work, we include the adsorption geometry of DIP [32] and PTCDA on Au(111) [24], $\mathrm{Ag}(111)$ [22] and on $\mathrm{Cu}(111)$ [23]. The adsorption distances on $\mathrm{Au}(111)$ have been corrected taking into account the surface reconstruction [24].

ordering. A similar argument was made for strongly interacting PTCDI on $\mathrm{Si}(111)$ [37] where near-edge x-ray fine structure (NEXAFS) spectroscopy measurements showed a lack of molecular order and a remarkable distortion in the first layer.

Having established the valence electronic structure and lateral as well as vertical order, we now turn to the corelevel analysis. Considering the chemical structure of the free molecule, PTCDI has four main inequivalent carbon atoms, three in the perylene core $(\mathrm{C}-\mathrm{C}, \mathrm{C}-\mathrm{H}, \mathrm{C}-\mathrm{C}-\mathrm{O})$ and one in the functional group $(\mathrm{C}=\mathrm{O})$, whereas the four oxygen atoms as well as the two nitrogen atoms are equivalent. Hence one expects a single symmetric $\mathrm{N} 1 s$ and $\mathrm{O} 1 s$ core-level peak. This is clearly seen in the multilayer regime shown in Fig. 5(a) corresponding to $\sim 10 \mathrm{ML}$ deposited on $\mathrm{Au}(111)$, where nitrogen and oxygen present a single symmetric peak with a shake-up satellite [20]. Carbon shows two main peaks, the most intense one corresponds to carbon atoms in the perylene core, whereas the peak attributed to the functional group [20,38] is shifted by $\sim 3 \mathrm{eV}$ towards higher binding energies (BE). The spectra on different substrates have the same shape, but are rigidly shifted in $\mathrm{BE}$ according to the VL difference between films [see Fig. 1(c)].

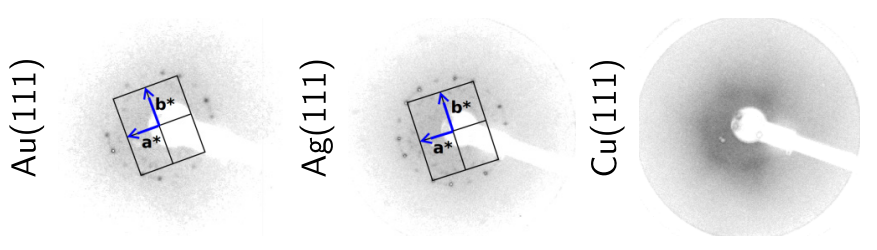

FIG. 4. LEED patterns for $1 \mathrm{ML}$ ( $4 \AA$ ) PTCDI, the unit cell is superimposed. Electron energies: $E=51 \mathrm{eV}$ for $\mathrm{Au}(111), E=55 \mathrm{eV}$ for $\mathrm{Ag}(111)$, and $E=29 \mathrm{eV}$ for $\mathrm{Cu}(111)$.

A key question is now whether the different adsorption scenarios can be connected to the (sub)monolayer core-level features displayed in Fig. 5(b), especially the interplay between the lateral and vertical interactions. For physisorbed PTCDI on $\mathrm{Au}(111)$, no preferential bonding with the substrate occurs through the functional groups. This is seen in the carbon HRXPS spectrum, where the shape is identical to the multilayer, and even the relative $\mathrm{BE}$ between perylene core and functional group peaks $(\sim 3 \mathrm{eV})$ remains unchanged. In the monolayer regime, though, the whole spectrum is rigidly shifted towards lower BE by $\sim 0.45 \mathrm{eV}$, which is typical for physisorbed molecules [21]. Actually, the rigid shift extends to the oxygen

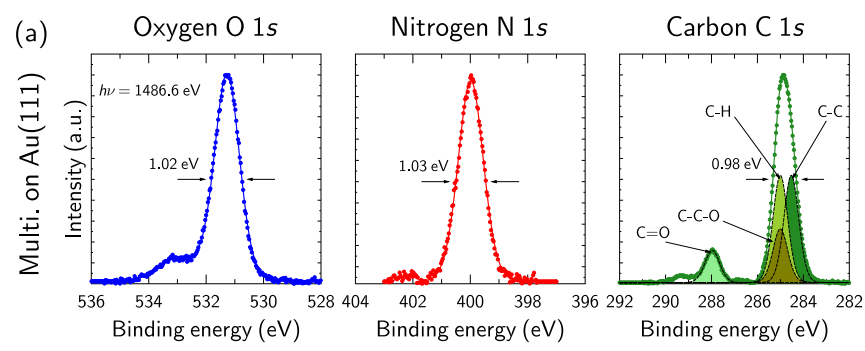

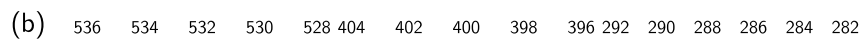

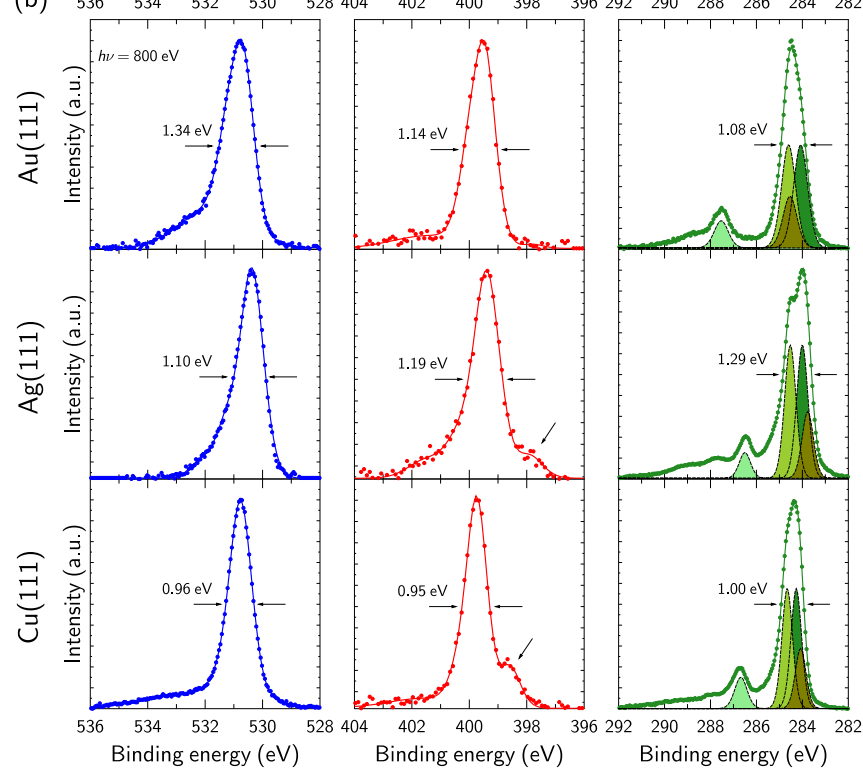

FIG. 5. XPS spectra of PTCDI at the multilayer (a) and (sub)monolayer regime (b). The inequivalent carbon atoms are assigned different components based on Refs. [20,28]. Shake-up structure has been omitted for clarity. (a) Multilayer deposited on $\mathrm{Au}(111)$ (monochromatic x-ray laboratory source). (b) HR-XPS scans $(h v=800 \mathrm{eV})$ for (sub)monolayer coverages. The widths of the main peaks are included. 


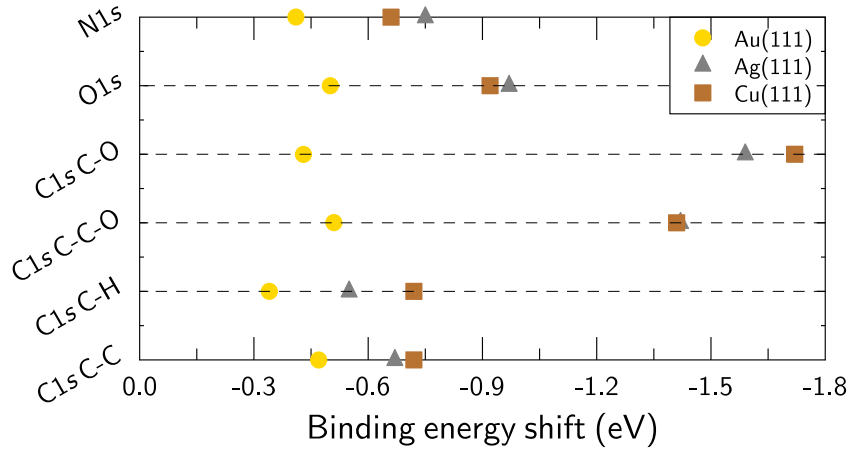

FIG. 6. Core-level shifts with respect to the multilayer BE for the different components at the (sub)monolayer regime. One can clearly see that on $\mathrm{Au}(111)$ the $\mathrm{BE}$ rigidly shifts by $\sim-0.45 \mathrm{eV}$, whereas on $\mathrm{Cu}(111)$ and $\operatorname{Ag}(111)$ the major shift occurs for the carbon atoms close to the functional groups.

and nitrogen core levels as displayed in Fig. 6. However, the line shape for these signals deviates significantly from the multilayer regime. First, the shake-up satellites move closer to the main line, attributed also to a larger screening efficiency of the core-hole by the substrate; and secondly and more importantly, these peaks become broader and slightly asymmetric, two effects that will be considered below.

As discussed by Häming et al. [38], the metallic character of the first PTCDI layer on $\mathrm{Ag}(111)$ has an impact on the core-level spectra. In particular, the high $\mathrm{BE}$ edge for the $\mathrm{C} 1 \mathrm{~s}$, $\mathrm{O} 1 s$, and $\mathrm{N} 1 s$ peaks exhibits an extended asymmetric tail, in remarkable contrast to the multilayer spectra in Fig. 5(a) but also to the same spectra on gold. The asymmetry stems from the continuum of interface states appearing upon hybridization of the molecular orbitals with the metal atoms [38], also responsible for the overall increase in intensity of the shake-up structure. The lower adsorption distance of the functional group causes the $\mathrm{C}=\mathrm{O}$ peak to move closer in $\mathrm{BE}$ to the main line. As seen in Fig. 6, the $\mathrm{C}=\mathrm{O}$ component shows a shift of $\sim 1.7 \mathrm{eV}$ towards lower $\mathrm{BE}$ with respect to the multilayer regime, i.e., $\sim 1.1 \mathrm{eV}$ more compared to $\mathrm{C}-\mathrm{C}$ and $\mathrm{C}-\mathrm{H}$, meaning that the benefit from the substrate screening is higher for the carbon atoms with electron deficiency (due to the bond with the more electronegative oxygen and nitrogen atoms). This trend extends to the $\mathrm{C}-\mathrm{C}-\mathrm{O}$ carbon atoms, as seen in the fits of Fig. 5, where this component experiences a strong shift towards lower $\mathrm{BE}$ and is responsible for the split of the perylene-core peak, similar to what it is reported for PTCDA on $\mathrm{Ag}(111)$ [28].

The core-level spectra corresponding to PTCDI deposited on $\mathrm{Cu}(111)$ show a decrease of the linewidth and an increased peak symmetry, in particular for nitrogen and oxygen. The BE shift in the $\mathrm{C} 1 s \mathrm{XP}$ spectrum with respect to the multilayer follows a similar trend as for silver (see Fig. 6), which agrees with the bending of the functional group towards the surface. Interestingly, the even shorter adsorption distance does not produce any significant improvement of the substrate screening compared to silver. Finally, we note the presence of a low BE peak [marked with an arrow in Fig. 5(b)] seen for $\mathrm{N} 1 s$ on $\mathrm{Cu}(111)$, less pronounced for $\mathrm{Ag}(111)$, but also reported for semiconductor substrates [39]. Its origin appears controversial. On $\operatorname{Ag}(111)$, the most intense peak is attributed to a giant satellite, whereas the low BE shoulder is considered the main line [38]. On the contrary, for PTCDI on $\mathrm{TiO}_{2}$, the low $\mathrm{BE}$ peak is attributed to nitrogen atoms that lose the hydrogen (deprotonation) due to substrate CT [39].

The different core-level features discussed so far could be unambiguously connected to the substrate influence. However, it does not explain the broader peaks seen on $\mathrm{Au}(111)$ compared to $\mathrm{Cu}(111)$. Indeed, the lifetime broadening increases with decreasing adsorption distance $[5,40]$, and our data show the opposite trend to what has been reported for other systems $[7,40,41]$. Because of the missing supramolecular arrangement of PTCDI on $\mathrm{Cu}(111)$ compared to $\mathrm{Au}(111)$, as inferred by LEED (see Fig. 4), and the enhanced intermolecular electronic coupling reported on the latter [36], we tentatively conclude that the asymmetry and broadening seen for the $\mathrm{N} 1 s$ and $\mathrm{O} 1 s$ core-level spectra on $\mathrm{Au}(111)$ is caused by the stronger molecule-molecule interaction, which is missing on $\mathrm{Cu}(111)$. It is reasonable to assume that PTCDI on $\mathrm{Ag}(111)$ represents an intermediate scenario, where the hybridization of molecular orbitals and the short adsorption distance (as seen in UPS and XSW) still leaves some freedom for the molecules to laterally rearrange (as shown in LEED), although the latter cannot be unambiguously assigned to any particular core-level feature beyond a reasonable broadening analogous to what happens on gold. Nonetheless, different studies of the similar PTCDA on various silver surfaces showed that molecule-molecule interactions have an effect on the frontier orbitals as seen by orbital tomography $[2,11]$. Also, the loss of lateral ordering upon cooling of a PTCDA monolayer on $\mathrm{Ag}(111)$ was shown to strengthen the bonding with the substrate, as indicated by a decrease in the adsorption distance, stronger bending of the functional groups and, most importantly, further filling of the LUMO [10], similar to what we observe on copper.

Consequently, due to the presence of the imide groups that facilitate a directional and strong molecule-molecule interaction, fingerprints of this interaction can be observed in the core-level spectra. This is in stark contrast to other molecular films that lack this preferential interaction groups, such as phtalocyanines [7,40,41] or DIP [40,41].

The experimental data presented here yield a comprehensive characterization of the structural and electronic properties of a complex metal-organic interface, with particular emphasis on the substrate reactivity as a mediator between moleculemolecule and molecule-substrate interactions and their impact on core-level spectra. We expect that the results presented will provide reliable data to further test available adsorption models $[1,2]$, especially regarding the role of functional groups and intermolecular interactions on the interface electronics and multilayer properties. Moreover, comparing the adsorption distances of chemisorbed PTCDI, PTCDA [25], and DIP [33] on $\mathrm{Cu}(111)$ and physisorbed perylene [34] on the same substrate shows that the functional groups are crucial for the existence of CT, but not for a short adsorption distance. Thus, using the bonding distance as exclusive indicator for organic-metal interaction strength can be misleading without a proper VB characterization.

The authors thank Diamond Light Source for access to beamline I09 and staff members T.-L. Lee and D. McCue for excellent support during the beamtime. We thank A. Schöll 
for reading the manuscript and providing useful comments. We also thank S. Hirschmann for the purification of PTCDI, $\mathrm{T}$. Hosokai for providing the perylene and R. Ji for assistance during UPS, XPS, and LEED measurements. Financial support from an NSFC Research Fund for International Young Sci- entists (No. 11550110176), the Soochow University-Western University Center for Synchrotron Radiation Research, the Collaborative Innovation Center of Suzhou Nano Science \& Technology (NANO-CIC) and the DFG is gratefully acknowledged.
[1] G. Heimel, S. Duhm, I. Salzmann, A. Gerlach, A. Strozecka, J. Niederhausen, C. Bürker, T. Hosokai, I. Fernandez-Torrente, G. Schulze, S. Winkler, A. Wilke, R. Schlesinger, J. Frisch, B. Bröker, A. Vollmer, B. Detlefs, J. Pflaum, S. Kera, K. J. Franke, N. Ueno, J. I. Pascual, F. Schreiber, and N. Koch, Nat. Chem. 5, 187 (2013).

[2] M. Willenbockel, D. Lüftner, B. Stadtmüller, G. Koller, C. Kumpf, S. Soubatch, P. Puschnig, M. G. Ramsey, and F. S. Tautz, Phys. Chem. Chem. Phys. 17, 1530 (2014).

[3] X. Bouju, C. Mattioli, G. Franc, A. Pujol, and A. Gourdon, Chem. Rev. 117, 1407 (2017).

[4] B. Stadtmüller, S. Schröder, and C. Kumpf, J. Electron Spectrosc. Relat. Phenom. 204, 80 (2015).

[5] E. Goiri, P. Borghetti, A. El-Sayed, J. E. Ortega, and D. G. de Oteyza, Adv. Mater. 28, 1340 (2016).

[6] A. El-Sayed, P. Borghetti, E. Goiri, C. Rogero, L. Floreano, G. Lovat, D. J. Mowbray, J. L. Cabellos, Y. Wakayama, A. Rubio, J. E. Ortega, and D. G. de Oteyza, ACS Nano 7, 6914 (2013).

[7] P. Borghetti, A. El-Sayed, E. Goiri, C. Rogero, J. Lobo-Checa, L. Floreano, J. E. Ortega, and D. G. de Oteyza, ACS Nano 8, 12786 (2014).

[8] A. Kraft, R. Temirov, S. K. M. Henze, S. Soubatch, M. Rohlfing, and F. S. Tautz, Phys. Rev. B 74, 041402(R) (2006).

[9] N. Gonzalez-Lakunza, M. E. Cañas-Ventura, P. Ruffieux, R. Rieger, K. Müllen, R. Fasel, and A. Arnau, Chem. Phys. Chem. 10, 2943 (2009).

[10] L. Kilian, A. Hauschild, R. Temirov, S. Soubatch, A. Schöll, A. Bendounan, F. Reinert, T.-L. Lee, F. S. Tautz, M. Sokolowski, and E. Umbach, Phys. Rev. Lett. 100, 136103 (2008).

[11] M. Willenbockel, B. Stadtmüller, K. Schönauer, F. C. Bocquet, D. Lüftner, E. M. Reinisch, T. Ules, G. Koller, C. Kumpf, S. Soubatch, P. Puschnig, M. G. Ramsey, and F. S. Tautz, New J. Phys. 15, 033017 (2013).

[12] C. Huang, S. Barlow, and S. R. Marder, J. Org. Chem. 76, 2386 (2011).

[13] M. C. R. Delgado, E.-G. Kim, D. A. d. S. Filho, and J.-L. Bredas, J. Am. Chem. Soc. 132, 3375 (2010).

[14] M. Bedzyk, in Encyclopedia of Condensed Matter Physics (Elsevier, Oxford, 2005), Vol. 6, pp. 330-341.

[15] C. Bürker, A. Franco-Cañellas, K. Broch, T.-L. Lee, A. Gerlach, and F. Schreiber, J. Phys. Chem. C 118, 13659 (2014).

[16] A. Yang, A. Franco-Cañellas, M. Sato, B. Wang, R.-B. Wang, H. Koike, I. Salzmann, P. K. Thakur, T.-L. Lee, L. Liu, S. Kera, A. Gerlach, K. Kanai, J. Fan, F. Schreiber, and S. Duhm, Phys. Rev. B 94, 155426 (2016).

[17] M.-C. Lu, R.-B. Wang, A. Yang, and S. Duhm, J. Phys.: Condens. Matter 28, 094005 (2016).
[18] See Supplemental Material at http://link.aps.org/supplemental/ 10.1103/PhysRevMaterials.1.013001 for a detailed explanation of the experimental conditions and the XSW technique. Also included are all values extracted from the measurements, together with additional XPS and UPS data for PTCDI and the XSW of unsubstituted perylene.

[19] F. S. Tautz, Prog. Surf. Sci. 82, 479 (2007).

[20] A. Schöll, Y. Zou, M. Jung, T. Schmidt, R. Fink, and E. Umbach, J. Chem. Phys. 121, 10260 (2004).

[21] Y.Zou, L. Kilian, A. Schöll, T. Schmidt, R. Fink, and E. Umbach, Surf. Sci. 600, 1240 (2006).

[22] A. Hauschild, K. Karki, B. C. C. Cowie, M. Rohlfing, F. S. Tautz, and M. Sokolowski, Phys. Rev. Lett. 94, 036106 (2005).

[23] A. Gerlach, S. Sellner, F. Schreiber, N. Koch, and J. Zegenhagen, Phys. Rev. B 75, 045401 (2007).

[24] S. K. M. Henze, O. Bauer, T.-L. Lee, M. Sokolowski, and F. S. Tautz, Surf. Sci. 601, 1566 (2007).

[25] S. Duhm, A. Gerlach, I. Salzmann, B. Bröcker, R. Johnson, F. Schreiber, and N. Koch, Org. Electron. 9, 111 (2008).

[26] O. Bauer, G. Mercurio, M. Willenbockel, W. Reckien, C. H. Schmitz, B. Fiedler, S. Soubatch, T. Bredow, F. S. Tautz, and M. Sokolowski, Phys. Rev. B 86, 235431 (2012).

[27] G. Mercurio, O. Bauer, M. Willenbockel, N. Fairley, W. Reckien, C. H. Schmitz, B. Fiedler, S. Soubatch, T. Bredow, M. Sokolowski, and F. S. Tautz, Phys. Rev. B 87, 045421 (2013).

[28] B. Stadtmüller, N. Haag, J. Seidel, G. van Straaten, M. Franke, C. Kumpf, M. Cinchetti, and M. Aeschlimann, Phys. Rev. B 94, 235436 (2016).

[29] J. C. Swarbrick, J. Ma, J. A. Theobald, N. S. Oxtoby, J. N. O'Shea, N. R. Champness, and P. H. Beton, J. Phys. Chem. B 109, 12167 (2005).

[30] M. Mura, F. Silly, G. A. D. Briggs, M. R. Castell, and L. N. Kantorovich, J. Phys. Chem. C 113, 21840 (2009).

[31] L. Romaner, G. Heimel, J. L. Brédas, A. Gerlach, F. Schreiber, R. L. Johnson, J. Zegenhagen, S. Duhm, N. Koch, and E. Zojer, Phys. Rev. Lett. 99, 256801 (2007).

[32] C. Bürker, N. Ferri, A. Tkatchenko, A. Gerlach, J. Niederhausen, T. Hosokai, S. Duhm, J. Zegenhagen, N. Koch, and F. Schreiber, Phys. Rev. B 87, 165443 (2013).

[33] K. Yonezawa, Y. Suda, S. Yanagisawa, T. Hosokai, K. Kato, T. Yamaguchi, H. Yoshida, N. Ueno, and S. Kera, Appl. Phys. Express 9, 045201 (2016).

[34] K. Manandhar and B. A. Parkinson, J. Phys. Chem. C 114, 15394 (2010).

[35] J. N. O'Shea, A. Saywell, G. Magnano, L. M. Perdigão, C. J. Satterley, P. H. Beton, and V. R. Dhanak, Surf. Sci. 603, 3094 (2009).

[36] J. Hieulle and F. Silly, J. Mater. Chem. C 1, 4536 (2013). 
[37] J. Taborski, P. Väterlein, H. Dietz, U. Zimmermann, and E. Umbach, J. Electron Spectrosc. Relat. Phenom. 75, 129 (1995).

[38] M. Häming, A. Schöll, E. Umbach, and F. Reinert, Phys. Rev. B 85, 235132 (2012).

[39] V. Lanzilotto, G. Lovat, G. Fratesi, G. Bavdek, G. P. Brivio, and L. Floreano, J. Phys. Chem. Lett. 6, 308 (2015).
[40] D. G. de Oteyza, I. Silanes, M. Ruiz-Osés, E. Barrena, B. P. Doyle, A. Arnau, H. Dosch, Y. Wakayama, and J. E. Ortega, Adv. Funct. Mater. 19, 259 (2009).

[41] D. G. de Oteyza, J. M. García-Lastra, M. Corso, B. P. Doyle, L. Floreano, A. Morgante, Y. Wakayama, A. Rubio, and J. E. Ortega, Adv. Funct. Mater. 19, 3567 (2009). 